\title{
GASP V: Ram-pressure stripping of a ring Hoag's-like galaxy in a massive cluster
}

\author{
A. Moretti, ${ }^{1 \star}$ B.M. Poggianti, ${ }^{1}$ M. Gullieuszik,${ }^{1}$ M. Mapelli, ${ }^{1}$ Y.L. Jaffé,${ }^{2}$ J. Fritz, ${ }^{3}$ \\ A. Biviano, ${ }^{4}$ G. Fasano, ${ }^{1}$ D. Bettoni, ${ }^{1}$ B. Vulcani, ${ }^{1,5}$ and M. D'Onofrio ${ }^{6}$ \\ 1 INAF-Astronomical Observatory of Padova vicolo dell'Osservatorio 5 35122 Padova, Italy \\ 2 European Southern Observatory, Alonso de Cordova 3107, Vitacura, Casilla 19001, Santiago de Chile, Chile \\ 3 Instituto de Radioastronomia y Astrofisica, UNAM, Campus Morelia, A.P. 3-72, C.P. 58089, Mexico \\ 4 INAF-Osservatorio Astronomico di Trieste, via G.B. Tiepolo 11, 34131 Trieste, Italy \\ 5 School of Physics, The University of Melbourne, Swanston St \& Tin Alley Parkville, VIC 3010, Australia \\ 6 Department of Physics and Astronomy, University of Padova, vicolo dell'Osservatorio 5, 35122 Padova, Italy
}

Accepted XXX. Received YYY; in original form ZZZ

\begin{abstract}
Through an ongoing MUSE program dedicated to study gas removal processes in galaxies (GAs Stripping Phenomena in galaxies with MUSE, GASP), we have obtained deep and wide integral field spectroscopy of the galaxy JO171. This galaxy resembles the Hoag's galaxy, one of the most spectacular examples of ring galaxies, characterized by a completely detached ring of young stars surrounding a central old spheroid. At odds with the isolated Hoag's galaxy, JO171 is part of a dense environment, the cluster Abell 3667, which is causing gas stripping along tentacles. Moreover, its ring counter-rotates with respect to the central spheroid. The joint analysis of the stellar populations and the gas/stellar kinematics shows that the origin of the ring was not due to an internal mechanism, but was related to a gas accretion event that happened in the distant past, prior to accretion onto Abell 3667, most probably within a filament. More recently, since infall in the cluster, the gas in the ring has been stripped by rampressure, causing the quenching of star formation in the stripped half of the ring. This is the first observed case of ram pressure stripping in action in a ring galaxy, and MUSE observations are able to reveal both of the events (accretion and stripping) that caused dramatic transformations in this galaxy.
\end{abstract}

Key words: surveys: GASP - galaxies: clusters: general - galaxies: evolution galaxies: interactions - galaxies: peculiar - galaxies: star formation

\section{INTRODUCTION}

The current understanding of galaxy formation and evolution links galaxy properties to both the original conditions in which galaxies formed, and the environment in which they are embedded.

According to the current cosmological paradigm, most galaxies in the Universe undergo at least an episode of accretion, or merger, or collision. Observational signatures of such events are transient features that make galaxies morphologically peculiar for a relatively short time, typically for a few tens of Myr to a few Gyr. A peculiar morphology is therefore a transient phase in the life of a galaxy.

Peculiar galaxies, such as ring galaxies and galaxies with counter-rotating components, can offer the key to understand the mechanisms at play in galaxy formation, reveal-

^ E-mail: alessia.moretti@oapd.inaf.it ing for example whether gas accretion and/or mergers are playing a major role in shaping galaxies properties.

Both rings and counter-rotation can be explained in terms of internal or external mechanisms. In fact, the presence of stellar and gaseous rings around galaxies can be due to different effects (Schweizer et al. 1987). Internal mechanisms, such as slow secular evolution, can produce rings as a consequence of the formation of a bar that dynamically induces the accumulation of material in correspondence to the Lindblad resonances, i.e. the regions where radial gas inflows are slowed down (Buta \& Crocker 1993; Heller \& Shlosman 1996). External mechanisms are related to environmental processes, such as major or minor mergers, or gas accretion either of low metallicity circumgalactic gas (Spavone \& Iodice 2013) or from a satellite galaxy (Buta \& Combes 1996).

Moreover, head-on collisions between a disk galaxy and a companion galaxy can produce the so-called collisional 
ring galaxies (the most famous example being the Cartwheel galaxy). In this case, the passage of the companion through the disk induces a perturbation in the orbits of the disk stars which propagates as a density wave (Mapelli et al. 2008). This implies that collisional rings are composed of stars that were already present in the disk galaxy, with no significant accretion from the companion galaxy. Moreover, the ring preserves the rotation of the original disk.

The idea of external gas accretion, while initially ruled out to explain ring galaxies due to the low frequency of isolated gas clouds with respect to the number of observed galaxies (Appleton \& Struck-Marcell 1996), has been recently reconsidered in the cold mode of gas accretion (Maccio' et al. 2006). Evidence for this mechanism is corroborated by observations of counter-rotation in some ringed galaxies (e.g. IC 2006 and NGC 77427, Sil'chenko \& Moiseev 2006). In particular, this scenario has been invoked to explain the origin of polar ring galaxies, i.e. galaxies that have two kinematically distinct components, inclined by almost 90 degrees to each other (Spavone et al. 2010), as well as the origin of rings highly inclined with respect to the host galaxy disk in general (Spavone \& Iodice 2013).

Some of the mechanisms proposed for the formation of ring galaxies have been invoked also for the existence of counter-rotating components in galaxies (Bettoni et al. 2001; Mapelli et al. 2008). Theoretical studies have shown that both a prolonged gas infall and a merger with a dwarf galaxy can lead to the observed counter-rotation (Jore et al. 1996; Thakar et al. 1996). In particular, in the merger case, the end product is an undisturbed optical morphology if the merger occurred more than 1 Gyr before the observations and, therefore, it would be accompanied by $>1$ Gyr old stars in the counter-rotating component.

The formation of counter-rotating gaseous disks seems to be favored in gas-poor systems, like S0 galaxies, while in spiral disks, hosting large amounts of gas co-rotating with the stellar component, is less frequent (Bettoni et al. 2014). In fact, a counter-rotating gaseous disk will be observed only if the mass of the newly supplied gas exceeds that of the pre-existing one (Lovelace \& Chou 1996). As for the stellar component, the counter-rotating components detected in samples of S0 galaxies (Johnston et al. 2012; Coccato et al. 2015; Katkov et al. 2013) have younger stellar populations compared to the main stellar disks, and their ionized gas rotates in the same direction as the secondary stellar components, i.e. it also counter-rotates with respect to the main disk. The youngest counter-rotating stars, therefore, should have been formed from the externally accreted gas. Evidence in favor of this interpretation comes also from recent results obtained with integral field spectroscopy of blue galaxies in the MaNGA survey (Chen et al. 2016).

One clear example of a non-collisional ring galaxy is the Hoag's object, an isolated galaxy at redshift 0.04 consisting of a central spheroid surrounded by a completely detached ring of young stars and gas. The absence of a central bar, as well as of an inclined disk, together with the strong contribution of the external ring to the total light of the galaxy (at least $20 \%$ in the case of the Hoag's object) are its main characteristics, and led Schweizer et al. (1987) to use these features to define a class of objects, called Hoagtype galaxies, which are neither obviously barred nor obviously inclined disks, and which have outer rings containing a significant fraction of the total luminosity. The presence of a dissolved bar was invoked as the origin of the ring in the Hoag's galaxy by Brosch (1985). However, subsequent studies revealed that the core of this peculiar galaxy is a spheroidal object, and not a disk, thus favoring a scenario where the ring was formed due to an accretion event 2-3 Gyr ago (Schweizer et al. 1987; Finkelman et al. 2011). Integral field spectroscopy of the Hoag's galaxy (Finkelman et al. 2011) confirmed that the central spheroid is consistent with being a classical bulge, typical of an elliptical galaxy. Its age could be as old as 10 Gyr, while the ring's age is younger than 2 Gyr. Stars in the central spheroid and in the ring share the same kinematics, i.e. rotate in the same direction.

In what follows we present MUSE integral-field spectroscopy of JO171, a peculiar galaxy that resembles the Hoag's galaxy and is undergoing strong ram pressure stripping in a massive galaxy cluster. We will discuss how its stellar and gas properties can be explained, searching for the origin of its doubly peculiar characteristics. After having presented our observations and methods $(\S 2)$, we will show the results regarding the structure of JO171 (§3.1), its gas and stellar kinematics (§3.2), stellar population ages ( $§ 3.3)$ and environment ( $\S 3.4$ ), discussing the formation and evolution of this galaxy in $\S 4$ and summarizing our findings in $\S 5$. Throughout this paper, we use a standard concordance cosmology with $H_{0}=70 \mathrm{~km} \mathrm{~s}^{-1} \mathrm{Mpc}^{-1}, \Omega_{M}=0.3$ and $\Omega_{\Lambda}=0.7$ and a Chabrier 2003 IMF.

\section{OBSERVATIONS AND METHODS}

In optical images, JO171 $(\mathrm{RA}=20: 10: 14.6, \quad \mathrm{DEC}=-$ 56:38:20.7) shows a disturbed morphology with one-sided tails suggestive of stripping by the Intra Cluster Medium (ICM). It was therefore been tagged as a candidate "jellyfish" galaxy by Poggianti et al. (2016). Jellyfish galaxies are those exhibiting ongoing star formation occurring in tails of stripped gas (Smith et al. 2010).

JO171 is a member of the cluster Abell 3667 (A3667), surveyed by the WIde Field Nearby Galaxy-Cluster Survey (WINGS, Fasano et al. 2006; Moretti et al. 2014) and its extension OmegaWINGS (Gullieuszik et al. 2015; Moretti et al. 2017). Its WINGS ID is WINGSJ201014.69-563830.1 and its spectroscopic redshift is 0.052529 . At this redshift 1 arcsec corresponds to $1.01 \mathrm{kpc}$.

This galaxy is part of the GASP survey (Gas Stripping Phenomena with MUSE, Poggianti et al. 2017), dedicated to study jellyfish galaxies across a wide range of galaxy masses and environments. GASP uses the Integral Field Spectrograph MUSE at the Very Large Telescope (VLT) (Bacon et al. 2010), which is capable of obtaining a spectrum for each of the $\sim 90000$ spaxels covering the $1^{\prime} \times 1^{\prime}$ field of view. The MUSE datacube has a spectral sampling of $1.25 \AA /$ pix, a spatial sampling of $0.2 \mathrm{arcsec} / \mathrm{pix}$ and a spectral range between 4500 and $9300 \AA$ with a spectral resolution of $\sim 2.6$. JO171 was observed with MUSE on 13 May 2016, with a seeing of 0.99 arcsec, for a total exposure time of $2700 \mathrm{sec}$ split into three $675 \mathrm{sec}$ exposures. The data were reduced using the ESO MUSE pipeline following the standard GASP procedure fully described in Poggianti et al. (2017).

From the MUSE datacube we measured the gas kinematics by fitting the emission lines ( $\mathrm{H} \alpha, \mathrm{H} \beta,[\mathrm{NII}],[\mathrm{OIII}])$, 
using the KUBEVIZ software (Fossati et al. 2016). The velocity of each spaxel in the frame is derived with respect to a given redshift, that is the redshift of the spheroid. Among all the emission lines used by KUBEVIZ the most prominent ones are the triplet around $\mathrm{H} \alpha$ comprising also the Nitrogen lines. This triplet has been fitted with a common continuum and variable fluxes. The continuum is calculated as the mean value calculated inside two windows redwards and bluewards of each line between 80 and $200 \AA$, and using values between 40 th and 60 th percentiles. The ratio between the two [NII] and [OIII] lines has been kept constant in the fit assuming the ratios given in Storey \& Zeippen (2000). Measurements have been made on a mean filtered $5 \times 5$ pixels kernel datacube, in order to maximize the signal to noise.

We determined the stellar kinematics using the Penalized Pixel-Fitting (pPXF) software (Cappellari \& Emsellem 2004) on spatially binned spectra. For the binning we used a Voronoi tessellation algorithm (Cappellari \& Copin 2003) that analyzes the white light image extracted from the original datacube and bins spectra on the basis of their signalto-noise $(\mathrm{S} / \mathrm{N})$. We imposed a $\mathrm{S} / \mathrm{N}=10$. The derived spectra have been fitted with the Vazdekis et al. (2010) stellar population templates. In particular we used Single Stellar Populations (SSP) with metallicities that range from $[\mathrm{M} / \mathrm{H}]=-1.71$ to $[\mathrm{M} / \mathrm{H}]=0.22$ and ages from 1 to $17.78 \mathrm{Gyr}$, calculated with the Girardi et al. (2000) isochrones.

We used our spectrophotometric code SINOPSIS, recently modified to be able to work with observed datacubes (Fritz et al. 2017). The code fits the observed spectra with a combination of SSPs from Charlot \& Bruzual (in prep.) calculated with a Chabrier (2003) Initial Mass Function (IMF) between 0.1 and 100 solar masses. The range in metallicities goes from $\mathrm{Z}=0.0001$ to $\mathrm{Z}=0.04$. On this set of SSPs nebular emission has been added. The code is also able to properly handle spectra with superimposed gas and stellar components with different velocities, as the two redshifts are given as inputs to the code. The SINOPSIS code gives as output a best fit model cube, as well as a stellar only model cube, that is then subtracted from the original one to obtain an emission only cube, useful for the determination of emission line fluxes corrected for stellar absorption. Finally, it gives stellar masses, star formation histories and stellar ages (both luminosity and mass weighted) for each spaxel. Due to the characteristics of the SINOPSIS code, we calculated the total mass in a given spaxel as the sum of the masses in four main age bins, but neglecting the mass of stars older than 0.57 Gyr when the spectra had a global S/N lower than 3. In these cases, in fact, the code is forced to find a not meaningful solution that includes a low percentage of old stars. The contribution of young stars, instead, is taken into account, given the fact that it is estimated from the emission lines, which are more trustable features.

The gas metallicity has been derived using the line flux measurements made on the emission-only and dustcorrected spectra of the spaxels where the ionization is typical of HII regions. In particular the internal extinction correction due to the galaxy dust has been taken into account measuring the Balmer decrement in each spaxel, and assuming an intrinsic $\mathrm{H} \alpha / \mathrm{H} \beta=2.86$ and the Cardelli et al. (1989) extinction law. We then used the pyqz code (Dopita et al. 2013) to derive both the ionization parameter $q$ and the gas metallicity through the comparison with a set of photo- ionization models (MAPPINGS IV). In particular, we used the $[\mathrm{NII}] 6583 /[\mathrm{SII}] 6716,6731$ vs [OIII] $5007 /[\mathrm{SII}] 6716,6731$ indicator, given that the $[\mathrm{OII}] 3727$ doublet lies beyond the MUSE spectral range, and that $\mathrm{H} \beta$ is prone to uncertainties in the subtraction of the stellar contribution. The systematic errors introduced by modeling inaccuracies are usually estimated to be $\sim 0.1-0.15$ dex, whereas discrepancies of up to 0.2 dex exist among the various calibrations based on photoionization models (Kewley \& Ellison 2008).

\section{RESULTS}

\section{$3.1 \quad$ JO171 structure}

From the reduced datacube, we extracted images in three bands, namely the I band, the $\mathrm{H} \alpha$ emission including the underlying continuum in a $40 \AA$-wide band and the B band, and constructed the RGB image shown in the left panel of Fig.1. A grayscale image is shown in the right panel. There are clearly two prominent structures: a central round spheroid and an external ring detached from the spheroid. The external ring shows a spiral-like pattern, more evident than in other Hoag-type galaxies. The ring is traced by the stellar continuum (in grey/white in Fig.1) and only his northern half has prominent emission lines (in green in Fig.1). The central spheroid lacks ionized gas, but for a few small blobs seen south and west of the spheroid. Figure1 also shows clear signatures of extended tails of ionized gas towards the north, in the direction opposite to the cluster center, confirming that JO171 is a jellyfish galaxy, whose disturbed morphology is linked with the environmental conditions.

The left panel of Fig.2 shows the JO171's B band surface brightness profile together with the Hoag's galaxy surface brightness profile (in green, Finkelman et al. 2011). The JO171 profile has been obtained averaging over all directions after having masked both the stars and the regions characterized by very peaked star formation. For the sake of comparison, the surface brightness profiles have been normalized to the same central surface brightness. We also derived the surface brightness profiles in other bands (SDSS u-band from OmegaCAM images (D'Onofrio et al. in prep.), continuum under $\mathrm{H} \alpha$ and Cousin's I-band from the MUSE data, Johnson K-band from VIRCAM archive data) and found that the JO171 ring becomes fainter going towards longer wavelengths, showing that its light comes primarily from young stars.

The structural parameters have been measured using a bi-dimensional fit using the GALFIT tool (Peng et al. 2010). The central spheroid has been fitted with a Sersic law that has two free parameters: the Sersic index $\mathrm{n}$ and the effective radius $R_{e}$ that turned out to be $2.96 \pm 0.10$ and $1.39 \pm 0.06 \mathrm{kpc}$, respectively. The Sersic fit of the central spheroid is shown in red in the left panel of Fig.2. The surface brightness analysis led us to better define the two structures composing JO171: in the following, we call spheroid the region within a radius of $3 \mathrm{kpc}$, and ring the region extending from $6.5 \mathrm{kpc}$ (i.e. the distance at which the surface brightness profile starts to rise) out to the average distance where counts reach $3 \sigma$ above the background, that is at $\sim 20 \mathrm{kpc}$. We have marked the spheroid's effective radius and the limits of the two regions in the right panel of Fig.1. 

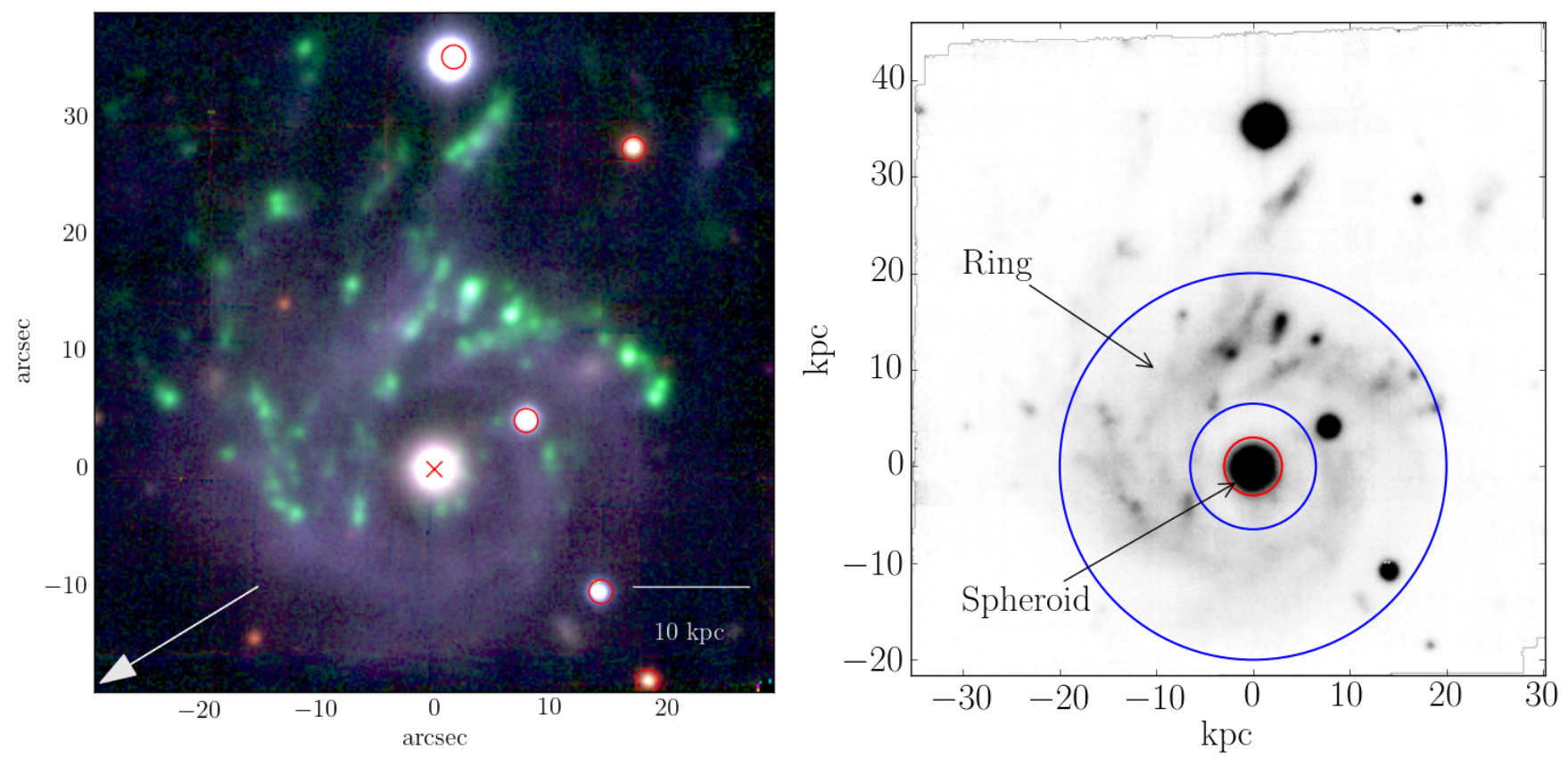

Figure 1. Left. RGB image of JO171 extracted from the MUSE datacube. The three bands correspond to the integrated flux in the Johnson I band (in grey colour), the integrated flux inside a $40 \AA$-wide band centered on H $\alpha$ (thus including both the H $\alpha$ emission and the continuum under $\mathrm{H} \alpha$, here in green color) and the integrated flux in the Johnson B band (blue in the image). Red circles indicate foreground stars that have been masked for the purposes of our analysis. The superimposed scale shows that $1^{\prime \prime}$ corresponds to 1.01 kpc at the galaxy redshift. North is up, East to the left. The arrow indicates the direction towards the Brightest Cluster Galaxy. Right. A grayscale image of the galaxy with superimposed a red circle enclosing the spheroid $\left(\boldsymbol{R}_{\boldsymbol{e}}\right)$, and blue circles enclosing the ring (see text).
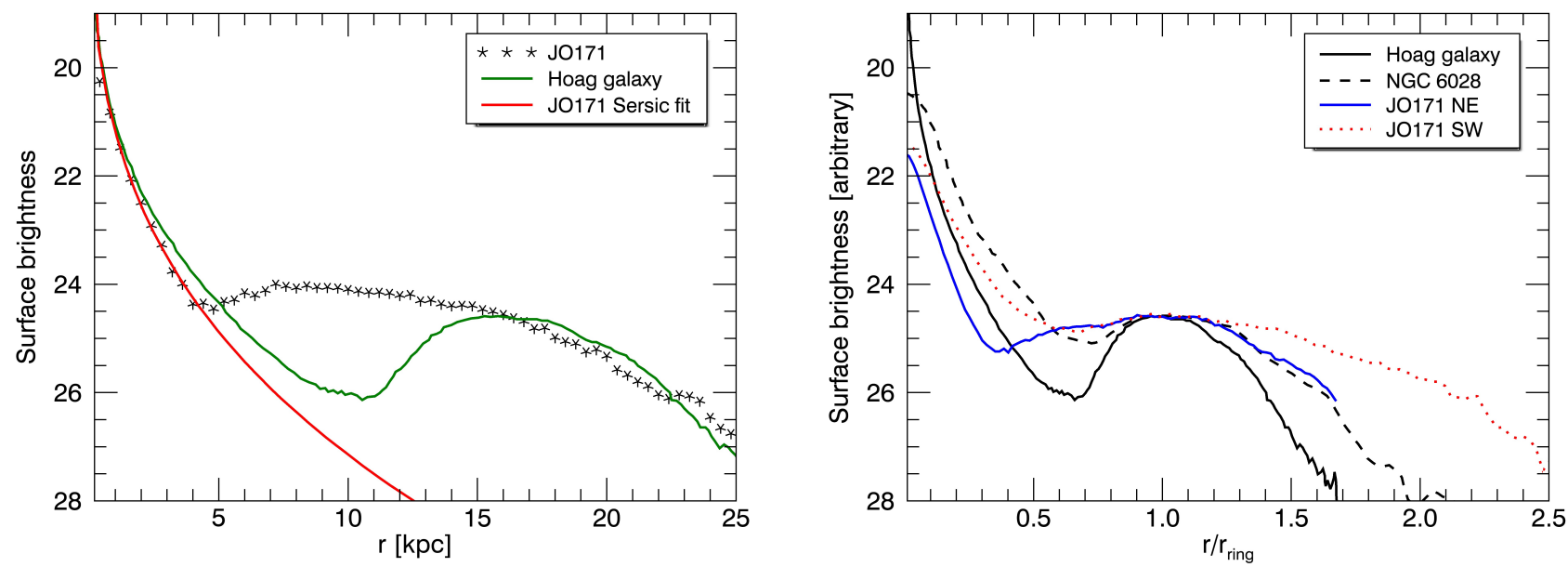

Figure 2. Left. Surface brightness profile of JO171 in mag arcsec ${ }^{-2}$, with superimposed the Sersic fit of the central spheroid in red. The green line is the Hoag's galaxy profile. Right. The blue and red profiles are the 180 degrees averaged profiles of JO171 north-east and south-west hemispheres, respectively. In black, the surface brightness profiles of the Hoag's galaxy (continuous line) and of the Hoag-type galaxy NGC 6028 (dashed line).

We integrated the MUSE spectra inside these two regions and fit them with the SINOPSIS code described in $\S 2$. The total galaxy mass of JO171 is $3.39 \pm 0.5 \times 10^{10} M_{\odot}$, with $56 \%$ of stars in the central spheroid, and $32 \%$ in the ring. The remaining mass is locked in the region between the spheroid and the ring (4\%) and in the stripped tails ( $8 \%)$.

Comparing with all WINGS clusters members with a known velocity dispersion (Fig.3), we find that the position of the JO171 spheroid in the various projections of the Fundamental Plane is perfectly in agreement with the locus occupied by elliptical/early-type galaxies, leading us to conclude that this is not a pseudo-bulge. Moreover, the spheroid characteristics $\left(v_{\max }=60 \mathrm{~km} \mathrm{~s}^{-1}, \sigma_{c} \sim 120 \mathrm{kms}^{-1}, e=0.05\right.$, where $\sigma_{c}$ is the central velocity dispersion and the ellipticity 

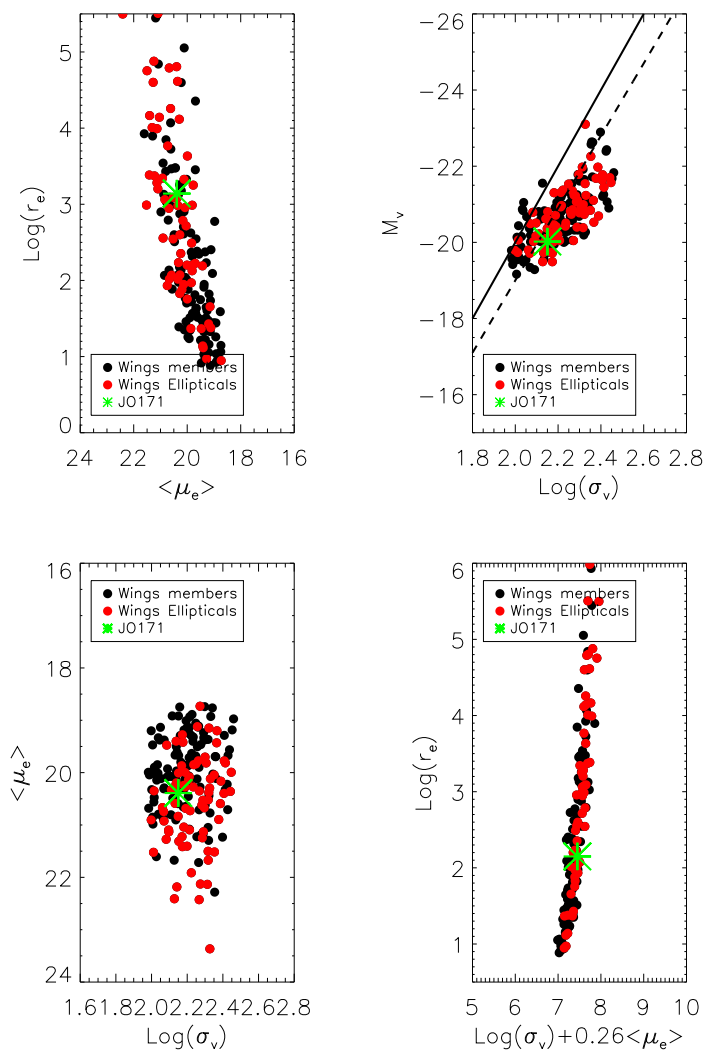

Figure 3. Fundamental Plane projections for WINGS cluster galaxies. In all panels the dots are spectroscopically confirmed cluster members, ellipticals (red) and S0s (black). The green asterisk is JO171. In the Faber -Jackson plane the continuous line shows the $L \propto \sigma^{4}$ relation, the dashed line a $L \propto \sigma^{3.8}$ relation.

is the average ellipticity within the spheroid) demonstrate that, similarly to the Hoag's central spheroid, this is a fast rotator, which indicates that the merging hypothesis for its formation is very unlikely.

The structure of JO171 looks similar to the Hoag's object: the two galaxies have a central spheroid of similar luminosity (-20.8, -20.7 in the B band, respectively), and the same effective radius of $\sim 2 \mathrm{kpc}$ (Finkelman et al. 2011). Both spheroids are almost round (ellipticity e $=0.03$ for Hoag's, 0.05 for JO171). The ring extends approximately between 12 and $25 \mathrm{kpc}$ in the Hoag's galaxy, and from 6.5 to $20 \mathrm{kpc}$ in JO171. We note that if we considered a ring extending out to $25 \mathrm{kpc}$ the results that we present in the following would not be altered. The mass of stars in the ring of Hoag's galaxy was estimated from its luminosity and simple assumptions on the stellar populations by Finkelman et al. (2011), and is $\sim 3 \times 10^{9} M_{\odot}$, smaller than that of JO171 $\left(1.1 \times 10^{10} M_{\odot}\right)$.

At odds with the Hoag's galaxy, JO171 shows extended tails towards the north characterized by ionized gas and ongoing star formation: while the Hoag's galaxy is isolated, JO171 belongs to a very active and rich galaxy cluster whose intracluster medium exerts a ram pressure force that creates the tails of stripped gas to the north.

As a consequence, its ring is clearly asymmetric in terms of the stellar populations/gas properties: the stripping of gas by ram pressure has caused the south-western part of the galaxy to be devoid of gas and, as we will see in $\S 3.3$, of ongoing star formation. This effect should be considered when comparing its surface brightness profile with the Hoag's galaxy and other Hoag-type galaxies. Ideally, we would like to analyze the JO171's profile before gas stripping began, and one way to approximate this situation is to consider the half of JO171 that has not been quenched/gas stripped yet, i.e. the north-eastern part.

In order to evaluate the differences between the starforming/gas-rich half of JO171's ring and the passive/gaspoor half of it, we draw a line inclined by 65 degrees N-W and calculate the 180 degrees average surface brightness profiles, that are shown in the right panel of Fig.2, together with the Hoag's galaxy and NGC 6028 profiles (black continuous and dashed lines, respectively): the blue line shows the starforming half of the ring, the red line the passive portion of it. In this figure we rescaled the radius to the ring radius and the surface brightness to the Hoag's galaxy ring surface brightness, in order to evaluate the magnitude dip between the spheroid and the ring in the different galaxies more easily.

It is clear from this figure that the profiles of the starforming and passive halfs of JO171 are different, and in particular that the north-east part of the ring shows a deeper gap between the spheroid and the ring. The magnitude difference is maximum for the Hoag's galaxy (1.5 magnitudes), and is minimum for the passive part of JO171's ring (0.3 magnitudes). NGC 6028 shows a dip of 0.5 magnitudes, while the star forming part of JO171's ring reaches 0.7 magnitude difference.

To conclude, JO171, as the Hoag's object and Hoagtype galaxies, is composed of two structures, a central round spheroid and a detached surrounding ring, whose origin will be investigated in the following sections.

\subsection{Gas and stellar kinematics}

From the MUSE datacube we measured the gas kinematics from the $\mathrm{H}_{\alpha}$ emission line and the stellar kinematics as described in $\S 2$. Formal errors in the velocity estimation of the gas component are below $3 \mathrm{~km} \mathrm{~s}^{-1}$ for most of the spaxels. The ring and the central spheroid share the same systemic velocity confirming that the two components belong to a unique system.

By comparing the ionized gas and stellar kinematics (Fig.4), we find that gas and stars of the ring share the same rotation, on average, but have quite different spatial distributions. While the gas extends only towards the North, stars are evenly distributed around the center. Gas and stellar velocities in the ring are similar, and reach a maximum value of $\sim 100 \mathrm{~km} \mathrm{~s}^{-1}$. The rotation axes of the two components are misaligned.

The gas tails and the fact that the stellar kinematics is instead undisturbed are clear signs that the gas is being stripped by a mechanism that affects the gas, but not the stars, i.e. ram pressure due to the interaction with the ICM. This is probably occurring approximately along the direction traced by the arrow in the top left panel of Fig.4 pointing towards the cluster BCG (Brightest Cluster Galaxy).

Interestingly, stars in the central spheroid counterrotate with respect to both stars and gas in the ring, with a rotation axis forming an angle of $\sim 225$ degrees, as can be 

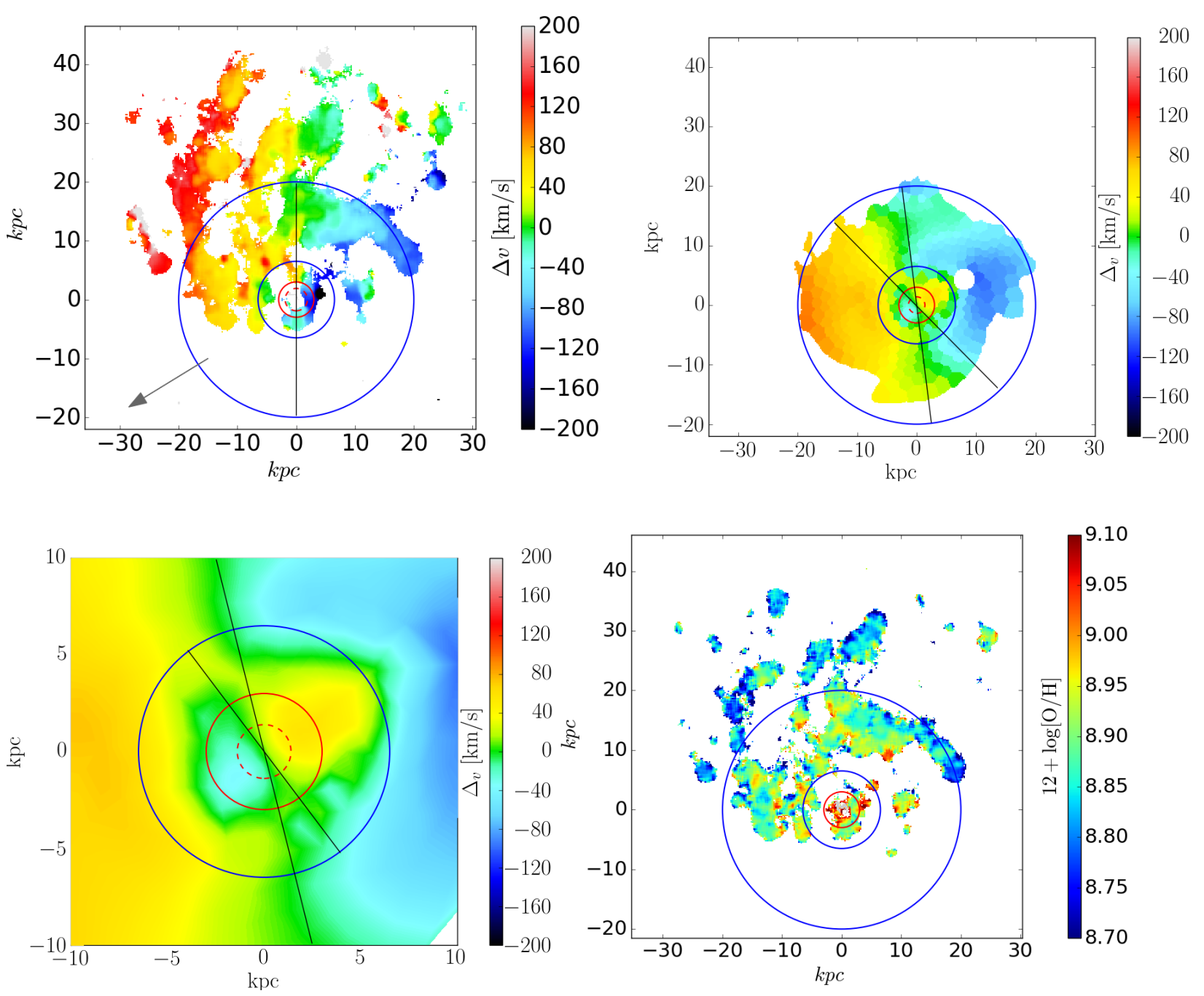

Figure 4. Gas (top left, $\mathrm{H} \alpha$ ) and stellar (top right) kinematics in JO171: ionized gas is present only in the northern part of the galaxy, and forms elongated tails that are being stripped from the galaxy. The arrow points toward the cluster center (i.e. the Brightest Cluster Galaxy, BCG). Stars are instead distributed in a symmetric pattern around the galaxy center. The solid red circle delimits the spheroid and corresponds to the red solid line in Fig.2, blue circles delimit the ring (also in blue in Fig.2). The almost vertical line is the rotation axis of the stars, while the second inclined line $\left(\sim 37^{\circ}\right)$ is the rotation axis of the central spheroid. The lower left panel shows a zoom in the central region of the stellar kinematics map, where the stellar spheroid counter-rotation appears. The bottom right panel shows the gas metallicity.

seen in the zoom shown in the bottom left panel of Fig.4. Here the maximum projected velocity is about $60 \mathrm{~km} \mathrm{~s}^{-1}$. The counter-rotation of the spheroid with respect to the ring is one of the major differences between JO171 and the Hoag's galaxy. We also note that the rotation axis of the spheroid is $\sim 37^{\circ}$ misaligned with respect to the one of the stellar ring.

The analysis of the kinematics clearly points towards an accretion/merger scenario, since (i) stars in the spheroid counter-rotate with respect to stars and gas in the ring, (ii) there is no evidence of a bar, and (iii) the rotational axis of stars in the ring and in the central spheroid are slightly misaligned.

The pure kinematical analysis cannot distinguish whether the accretion was due to the slow disruption of a mostly gaseous companion, or to cold accretion of gas along a filament, and it is not able to date the event itself, unless combined with the stellar population properties as done below.

\subsection{Stellar populations}

We further used the output from SINOPSIS to date the stellar populations and reveal the star formation history experienced by JO171. Fig.5 presents the logarithmic star formation rate density (in solar masses per year per squared $\mathrm{kpc}$ ) maps in four age bins characterizing ongoing star formation (stellar age $<2 \times 10^{7} \mathrm{yr}$ ), recent star formation (stellar ages between $2 \times 10^{7}$ and $<5.7 \times 10^{8} \mathrm{yr}$ ), intermediate age stars (between $5.7 \times 10^{8}$ and $5.7 \times 10^{9} \mathrm{yr}$ ) and old stars (older than $\left.5.7 \times 10^{9} \mathrm{yr}\right)$.

Globally, the fraction of present stellar mass (living 

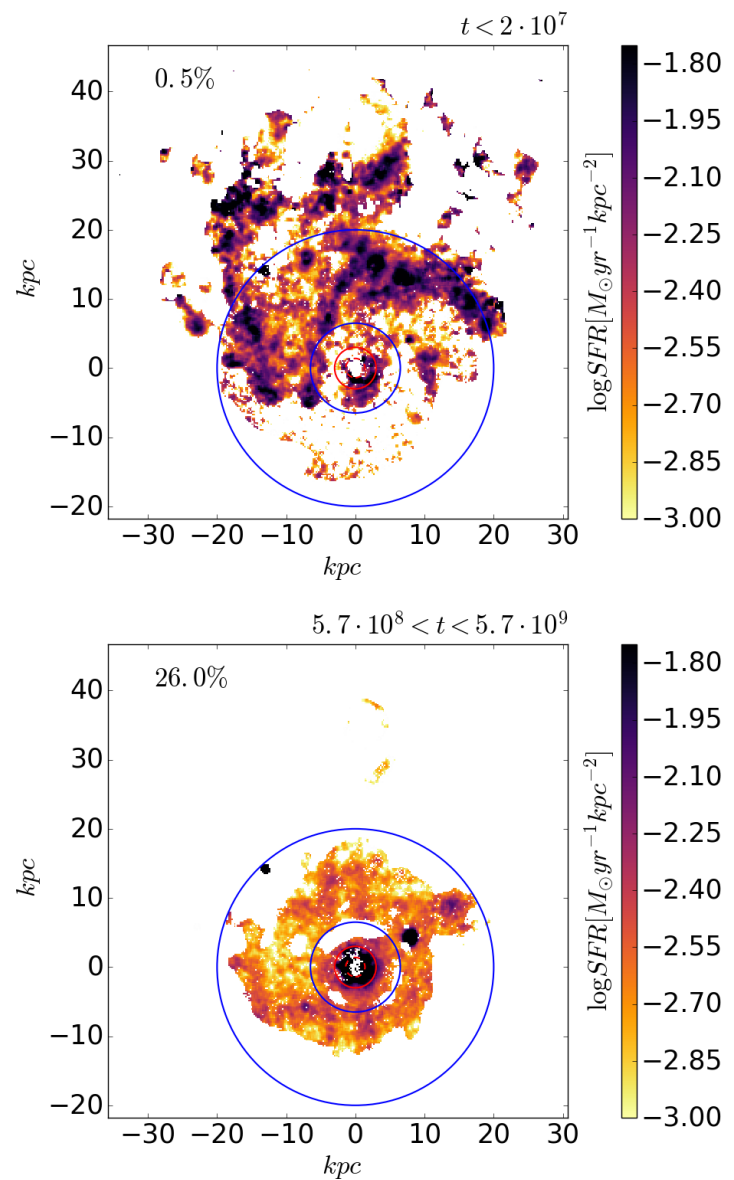
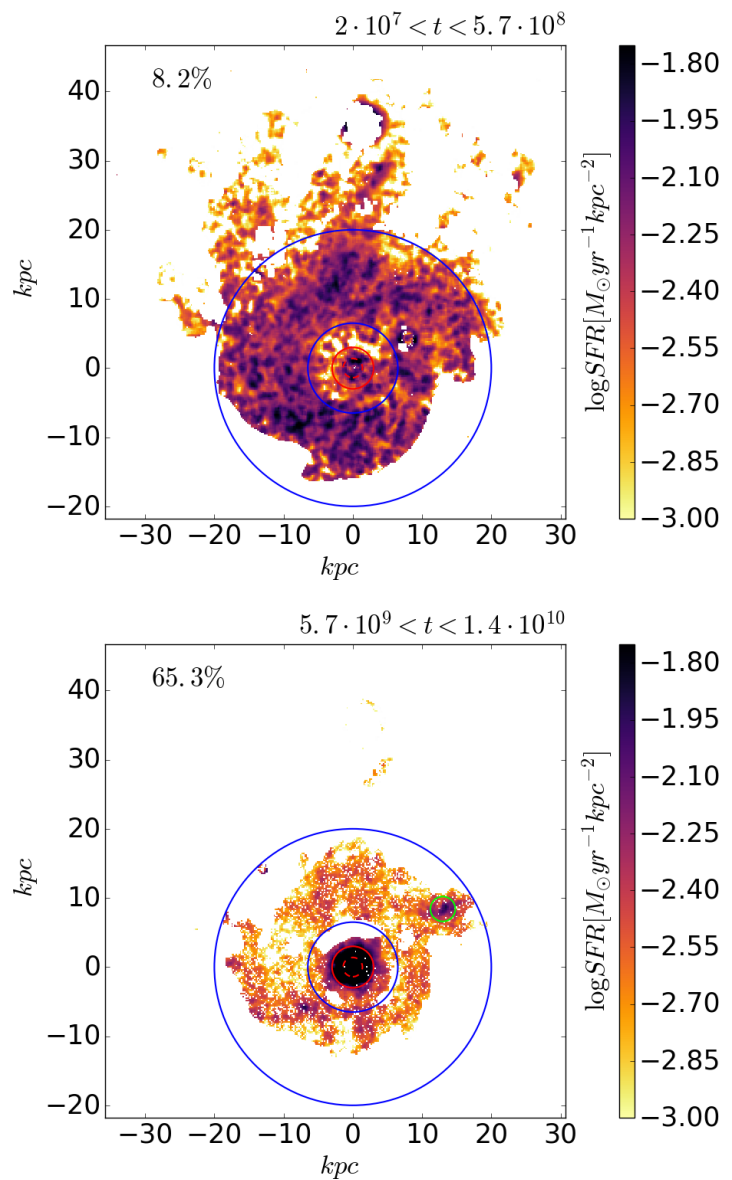

Figure 5. Star formation rates surface densities in 4 age bins as derived from SINOPSIS: from top left to bottom right we show the ongoing SFR (ages younger than $2 \times 10^{7} \mathrm{yr}$ ), the recent SFR (ages between $2 \times 10^{7}$ and $5.7 \times 10^{8} \mathrm{yr}$ ), intermediate and old SFRs (with ages between $5.7 \times 10^{8}$ and $5.7 \times 10^{9}$ and older than $5.7 \times 10^{9} \mathrm{yr}$, respectively). The green circle in the bottom right panel shows the blob of most enhanced star formation within the ring in the oldest age bin.

stars and remnants) formed in the four age bins is $0.5 \%$, $8.2 \%, 26.0 \%$ and $65.3 \%$ (from younger to older age bins) of the total stellar mass, i.e. about $91 \%$ of the total mass of the galaxy was formed before the last $0.6 \mathrm{Gyr}$.

Figure 5 shows that the star formation (SF) at early epochs was concentrated in the spheroid, even though a low level of star formation is seen also in the ring. The SF in the ring increases with time, peaking between $2 \times 10^{7}$ and $5.7 \times 10^{8} \mathrm{yr}$ ago, both in the Southern and Northern halfs of the ring. In contrast, the ongoing SF (ages $<2 \times 10^{7} \mathrm{yr}$ ) is occurring only where the ionized gas is still present, i.e. in the Northern half of the galaxy and in the tails.

In order to better understand the history of this galaxy, in Fig.6 we show the mass-age and star formation rate (SFR)-age relations. The left panel shows how the stellar mass was assembled over cosmic time in the spheroid (red asterisks), the ring (blue squares) and globally (black crosses). At the end of the oldest age bin, i.e. 6 Gyr ago, almost $85 \%$ of stars in the spheroid were already in place, while only $45 \%$ of the mass in the ring was born. About 0.6 Gyr ago the spheroid was almost totally formed (99\%) while the ring had $\sim 81 \%$ of its current mass. As better shown in the right panel of Fig. 6 (with the same color coding), the star formation histories of the spheroid and the ring are quite differ- ent: while the spheroid is characterized by a monotonously declining star formation, the ring sees its star formation significantly enhanced in the recent age bin (between 20 and 570 Myrs ago).

Overall, the analysis of the stellar population ages shows that 1) the central spheroid formed at early epochs and had a monotonously declining star formation rate; 2) the formation of the ring began during the oldest age bin, and the average SF of the ring was low and constant until $600 \mathrm{Myr}$ ago. We notice that the mass locked into old stars could be due either to a very low SFR protracted for a very long time or to single episodes of high SF, since the age resolution of spectrophotometric models is insufficient to age-date individual SF episodes within each age bin. 3) the average $\mathrm{SF}$ in the ring strongly increased during the last $600 \mathrm{Myr}$, forming $20 \%$ of its current mass in this period. In the next section, we will see that this increase is most likely a result of a SF enhancement due to the impact of the galaxy on the ICM when the galaxy entered the cluster.

\subsection{Environment}

JO171 is located in the cluster A3667, a very rich cluster with a virial mass $M_{200}=1.7 \times 10^{15} M_{\odot}$ (Moretti et al. 2017), 

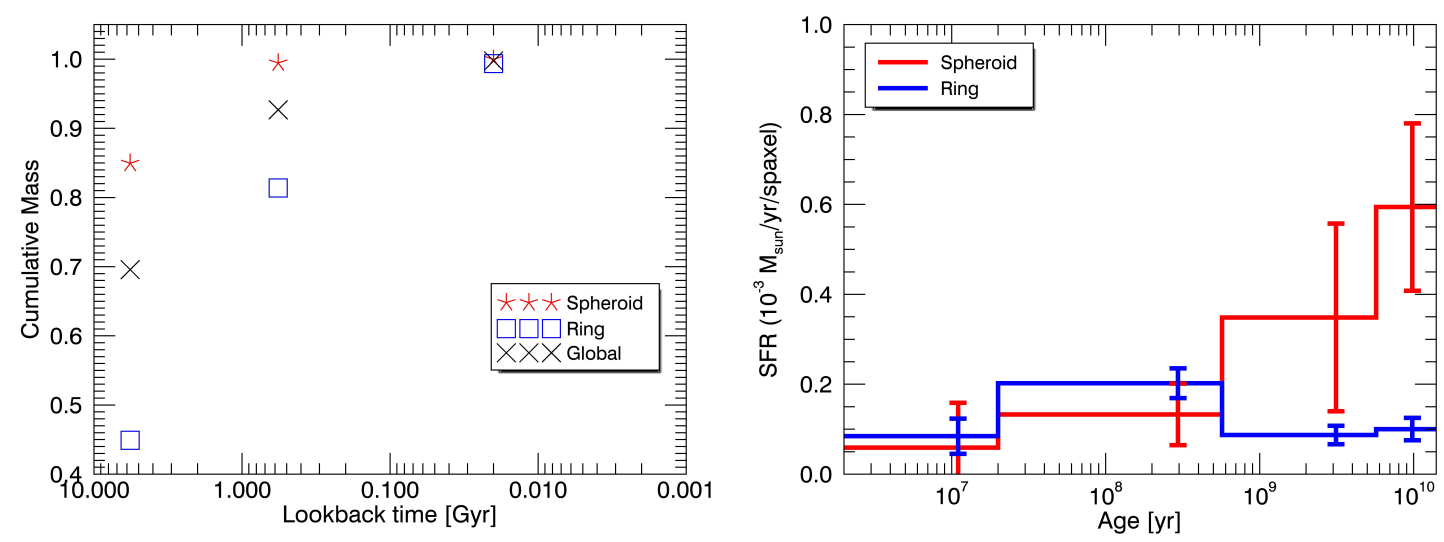

Figure 6. Cumulative mass-age (left) and (right) average star formation rate per spaxel-age relations for JO171: the central spheroid is traced by the red line/symbol and the ring by the blue. SFRs in the right panel are in units of $10^{-3}$ solar masses per year per spaxel.

that is dynamically very active: it possesses a cold gas front in the center (Vikhlinin et al. 2001) and two bright diffuse radio emission regions straddling the X-rays gas (Rottgering et al. 1997). A first analysis of cluster substructures (Owers et al. 2009) also suggests that A3667 is the result of two merging sub-clusters of similar masses in the plane of the sky, probably occurred $\sim 1$ Gyr ago. JO171 lies at a projected clustercentric distance of $\sim 0.64 R_{200}$ from the BCG, where $R_{200}$ is the radius at which the mean density of the cluster reaches 200 times the critical density and is expected to contain most of the virialized cluster mass.

A deeper understanding of the complex galaxy-cluster dynamical status could help linking the JO171's peculiar morphology to its position in the cluster. In particular, the dynamical analysis can help clarifying if the galaxy belongs to a substructure infalling into the cluster potential well or not. The left panel of Fig.7 shows the sky distribution of the spectroscopically confirmed cluster members, along with the identified substructures color-coded according to the probability of being randomly extracted from a Gaussian distribution with cluster typical values at the given distance. Galaxies plotted with bluest colors have a higher probability to belong to a substructure than the red ones. After having assigned cluster membership using the KMM algorithm to identify the main redshift peak, and then the Clean algorithm (Mamon et al. 2013) to refine it, the cluster substructures have been identified using all the spectroscopic information available from the OmegaWINGS spectroscopy (Moretti et al. 2017), complemented by literature data (Owers et al. 2009), using a dedicated algorithm described in Biviano et al. (2017).

While our algorithm does not assign JO171 (black star) to any substructure, the galaxy is located close to some identified substructures, so it is possible that there is also a physical - albeit undetected - relation with them. More likely, this peculiar galaxy was probably hit by a shock wave originated during the cluster merger, as suggested by its location close to a "radio bridge" identified NW of the cluster core (Carretti et al. 2012).

The right panel of Fig. 7 shows the projected clustercentric distance $r_{c l}$ plotted against the line-of-sight velocity relative to the cluster velocity $\Delta v_{c l}$. The resulting phasespace diagram (PPS) has been normalized by the cluster size and velocity dispersion. For reference, the escape velocity in a Navarro, Frenk \& White (Navarro et al. 1995) halo is shown by the grey curves. Cosmological simulations have shown that galaxies of different time since accretion into the cluster occupy different regions in projected phasespace, with the oldest cluster members concentrating at low $r_{c l}$ and low $\Delta v_{c l}$, while recent infalls having higher velocity dispersion and/or larger $r_{c l}$ (Rhee et al. 2017; Haines et al. 2015; Oman et al. 2013). The location of JO171 in phase-space is suggestive of recent accretion to the cluster, in agreement with the hypothesis that the recent burst of star formation in the ring is linked with the first impact with the cluster.

Since the work of Gunn \& Gott 1972 it is known that the ram pressure exerted by the hot ICM on infalling galaxies is proportional to the ICM density (that increases with decreasing $r_{c l}$ ), and the square of the galaxy peculiar velocity. The stripping predicted from this simplified analytical prescription coincides remarkably well with HI observations of cluster galaxies (Jaffe et al. 2015; Yoon et al. 2017). Highresolution cosmological hydrodynamic simulations of cluster galaxies indeed predict that galaxies can lose their cold gas during their first radial infall in the cluster (Cen et al. 2014). Simulations have also shown that an enhanced ram pressure efficiency can be the effect of ICM inhomogeneities, even in galaxies well beyond the virial radius (Tonnesen et al. 2007).

Assuming smooth ICM profiles, and Gunn \& Gott (1972)'s analytical prescription of ram pressure stripping, it is possible to predict the amount of gas stripping expected for infalling galaxies given their location in PPS diagrams. In short, stripping occurs when the intensity of ram pressure overcomes the anchoring force of the galaxy (Jaffé et al. submitted).

The anchoring force of JO171 is difficult to model since this galaxy has two components with different gas contents, and the gas in the ring is probably subject to a lower anchoring force than in a normal disk. Since JO171 peculiar morphology prevents a detailed modeling of the anchoring force, a conservative estimate of the amount of gas stripping 

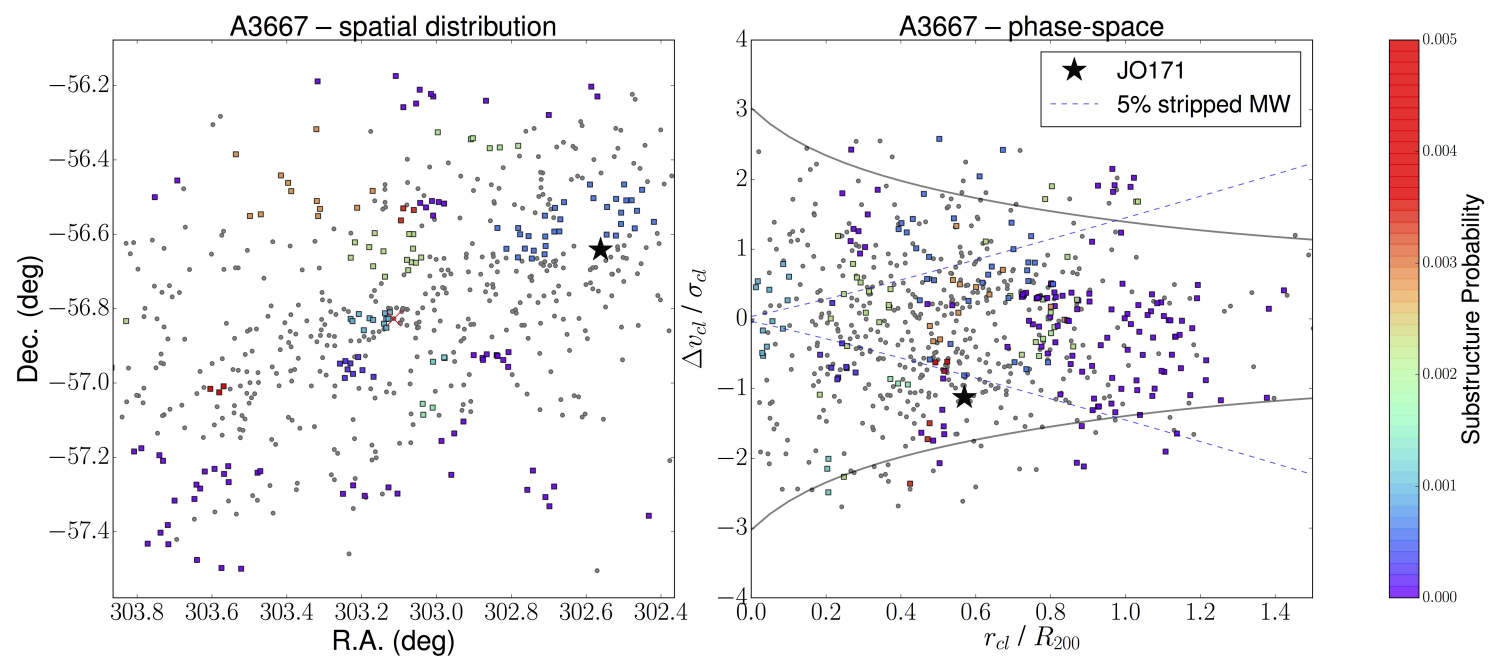

Figure 7. A3667 members and substructures: the left panel shows the spatial distribution of A3667 spectroscopic members (grey points) with superimposed the substructure members, color coded according to (1-p) where $\mathrm{p}$ is the probability to belong to a substructure. The right panel shows the same galaxies in the phase-space diagram. The black star shows the location of JO171. The dashed lines show the region where a Milky Way-like galaxy will lose $5 \%$ of its gas due to ram pressure stripping. The continuous lines show the escape velocity in a NFW halo.

can be obtained using as proxy a Milky Way-like disk galaxy in a cluster with a velocity dispersion $\sigma_{c l}=1000 \mathrm{~km} \mathrm{~s}^{-1}$, similar to the A3667 velocity dispersion $\left(1010 \mathrm{~km} \mathrm{~s}^{-1}\right)$, as in Jaffe et al. (2015). This method has already been used for GASP I (Poggianti et al. 2017), GASP II (Bellhouse et al. 2017) , GASP III (Fritz et al. 2017) and GASP IV (Gullieuszik et al. 2017) papers.

At the location of JO171 in the right hand plot of Fig.7, we estimate that an infalling Milky Way-like galaxy would have lost $5 \%$ of its total gas mass via ram-pressure (dashed blue lines, see simulations in Jaffé et al. submitted). This estimate is only a lower limit for the following reasons: i) we expect that the ring of JO171, being subject to a weaker anchoring force than the Milky Way, will suffer from a more intense RP, that will strip the gas earlier during infall (i.e. to the right of the dashed lines), ii) A3667 is a merging system with an inhomogeneous ICM that can cause enhancement of $\mathrm{RP}$, and iii) the measured velocity and distance of the galaxy from the cluster centre are projected values, and thus are lower limits to their real values. Given the morphology of the tails, JO171 is probably moving with respect to the cluster mostly within the plane of the sky, and the real velocity relative to the ICM will be much higher than the line-ofsight relative velocity observed.

In summary, the position of JO171 in phase-space indicates that the galaxy must be undergoing ram pressure stripping by the ICM, and both the presence of substructures in A3667 and the structural configuration of this galaxy suggest that the pressure exerted on JO171 is probably much higher than the one that can be computed with the standard assumptions.

\section{THE ORIGIN OF JO171}

Being a ring galaxy, JO171 offers the opportunity to study a secondary event in the lifetime of a galaxy, and the detailed analysis of its properties can cast light on the major/minor merger scenario as opposed to gas accretion. In addition, JO171 experienced a tertiary event, recently entering a rich cluster, and this gives us the unique opportunity to study the effects of ram pressure on a fragile ring galaxy.

Regarding the formation of the ring, our analysis seems to rule out an internal origin (bar induced resonances), given the absence of any bar signature in our data. Moreover, the small, but detectable, misalignment between the rotation axis of the stellar spheroid and the outer disk seems to confirm an external event in the galaxy's life.

We also discard the hypothesis that it is a collisional ring due to the passage of a small galaxy through the disk, as this scenario is unable to account for the formation of a counter-rotating component.

We are therefore left with only two scenarios: the gas rich merger and the cold accretion along a filament of intergalactic medium. Both scenarios have been invoked to explain the most massive polar rings (Bekki \& Kenji 1998; Iodice et al. 2006; Spavone \& Iodice 2013; Spavone et al. 2010), and evidence of gas accretion from either filaments or interactions with gas-rich satellites have been found in a few galaxies (Sancisi et al. 2008).

In the major merger case, numerical simulations (Jesseit et al. 2007) are able to produce an old and counter-rotating spheroid with a 1:1 retrograde merger only if including a $10 \%$ dissipative component. However, a certain amount of gas inflow toward the galaxy center is expected (Bournaud et al. 2007), and so is the subsequent episode of star formation. Moreover, models predict that the central galaxy will have a flattened luminosity profile, with ellipticity $\mathrm{e}=1-\mathrm{b} / \mathrm{a}$ reaching 0.5-0.6 after 4 merger events. Neither a significant young central stellar component, nor the flattening are observed in JO171.

The analysis of the structural properties of JO171 spheroid confirms that it is a fast rotator, therefore making the major merging hypothesis very unlikely. 
Simulations of minor mergers (Mapelli et al. 2015) are able to produce the observed long-lived star forming rings in many cases, but still induce a prolonged star formation episode in the central galaxy. However, in these simulations this effect is due to the presence of a bar in the main spheroid, that helps accumulating material in the central region. Our data, therefore, can not rule out a retrograde 1:2-1:5 merger as the origin of this galaxy. If the counterrotating ring is the result of a relatively minor merger, we might try to date the epoch of this merger based on the stellar populations.

Mergers with a gas-rich companion are known to trigger bursts of star formation. From Fig. 6 it is apparent that 45\% of the current stellar mass of the ring was already in place 6 Gyr ago.

If we attribute the last burst of SF $(\leq 0.5 \mathrm{Gyr}$ ago $)$ to the entrance of J0171 into the cluster and to the consequent RPS, this means that the merger must have happened long before the entrance of JO171 in the cluster. Vice versa, if we attribute the last burst of SF to the merger, we would date the first stages of the merger to $\sim 0.5$ Gyr ago. This would imply that the companion converted more than $81 \%$ of the current stellar mass of the ring into stars before merging with the spheroid, and that JO171 entered the cluster and underwent a merger approximately at the same time. However, this would also imply that the companion should have been disrupted in a very short timescale $(<0.5$ Gyr), without leaving any clear signatures of nucleus and tidal arms, which is unrealistic. Thus, if the ring of JO171 comes from a gas-rich merger, we expect that this merger happened more than 0.5 Gyr ago.

If this is what happened, we might be able to spot in the ring of the system faint signatures of the merger remnant. We identified in particular one region in the ring where the star formation rate in the oldest bin is enhanced (the green circle in the bottom right panel of Fig. 5), as a possible merger remnant, but its velocity turned out to be $\sim 1000$ $\mathrm{km} \mathrm{s}^{-1}$ larger than the galaxy one, thus excluding any link with JO171.

Regarding the possibility that the gas is accreted from a nearby companion,we verified that there are none within the MUSE field-of-view.

We also searched in our spectroscopic (> 90\% complete at $\mathrm{V}=20$ ) and photometric catalogs (Moretti et al. 2017; Gullieuszik et al. 2015) for possible companions and gas donors. Following the study by Brocca et al. (1997) we searched them within a region 5 times larger than the diameter of JO171's ring. We searched both galaxies with redshift brighter than $\mathrm{V}=20$, and any other candidate in the photometric catalog down to two magnitudes fainter than JO171. This search yields only two galaxies, both fainter than JO171 (1.4 and 2.6 mag fainter in B than JO171). They are both spectroscopically confirmed members lying at a projected distance of $\sim 90 \mathrm{kpc}$ from JO171, and have line-of-sight relative velocity of about 800 and $1350 \mathrm{~km} \mathrm{~s}^{-1}$ with respect to JO171. Given the high relative projected velocities we can exclude interactions among these galaxies, also given the fact that the close environment of JO171 does not seem to be perturbed at the present epoch. Moreover, none of the three galaxies (JO171, and the two highlighted galaxies) seems part of any substructure (see §3.4).

For all of these reasons, we consider the merger hy- pothesis the least likely, and the cold gas accretion along a filament hypothesis the most plausible scenario to explain JO171 properties.

Simulations (Agertz et al. 2009) predict that the accreted cold gas settles into a large disk-like system, which then fragments and forms stars in clumpy regions. In our case, this accretion should have started long before the galaxy accreted onto the cluster, as the old age of the ring stars demonstrates. Such rings can survive as long as 2-8 Gyrs (Maccio' et al. 2006), and have masses similar to the central spheroid.

In such a scenario, the metallicity of the gas in the ring is expected to be lower than the standard one for the galaxy total galaxy mass or luminosity. This result has already been confirmed in polar ring galaxies (Spavone et al. 2010).

After having removed the stellar component from the MUSE spectra, we derived the gas metallicity shown in Fig.4, bottom right panel. The gas has a median metallicity of $12+\log (\mathrm{O} / \mathrm{H}) \sim 8.87$, that is slightly lower than the expected 9.06 for a normal mass-metallicity relation (Tremonti et al. 2004) and lower than the average 9.1-9.2 value for its B-band luminosity (Spavone et al. 2010). This effect, however, is small for JO171, at the $2 \sigma$ level. This is because the ring and the spheroid have similar masses, while the effect should be much more visible if the ring were only a lower fraction of the total mass/luminosity. Moreover, the gas shows no hints for structured metallicity gradients, that would be expected in case of normal metal enrichment due to stellar populations aging in a disk galaxy.

Therefore, we propose a scenario of gas accretion at early epochs (first $\sim 6 \mathrm{Gyr}$ ), followed by a more recent star formation enhancement (as demonstrated by the higher SFR in the second youngest age bin) due to the interaction with the environment, at the beginning of the ram pressure effect. The ram pressure is also responsible for the complete stripping of the gas from the South region, and for the tentacles towards the North, where we observe the ongoing star formation.

\section{SUMMARY}

Within our ongoing ESO MUSE GASP Program, we have studied the characteristics and the evolutionary history of JO171, a peculiar $\sim 3 \times 10^{10} M_{\odot}$ ring galaxy in the dense environment of the cluster A3667. This galaxy resembles the well known Hoag's galaxy (Finkelman et al. 2011) and is constituted by a central spheroid surrounded by a ring of young stars/gas. Its ring gas is currently being stripped due to the ram-pressure exerted by the ICM on the infalling galaxy. Our data show that this galaxy underwent two transformations that dramatically changed the course of its evolution: the formation of the ring, most probably early on in its history, and the more recent stripping of its ring gas. It is the first time that a peculiar ring galaxy similar to the Hoag's object is observed in a dense cluster in the act of being transformed by environmental effects.

The availability of MUSE integral field spectroscopy allowed us to investigate its peculiar kinematics and to unravel the properties of its stellar populations with unprecedented details. As the Hoag's galaxy, JO171 does not show evidence for a central bar, strongly suggesting that the formation of 
the ring is due to an external cause. Moreover, at odds with the Hoag's galaxy, the central spheroid of JO171 counterrotates with respect to the ring, confirming that a secondary event must be at the origin of the ring.

JO171 currently displays long tails of ionized gas towards the north, while its southern half is totally devoid of gas. Both of these characteristics are due to ram pressure stripping acting approximately in the direction opposite to the cluster center.

The joint analysis of the stellar/gas kinematics and the stellar populations properties shows that, while the spheroid is generally old and characterized by a monotonously declining star formation history, the formation of the ring stars began early on, with an average SFR low and constant until $\sim 600$ Myr ago, then there was an increase in star formation activity during the last 500 Myr due to ram pressure upon cluster infall.

Both gas rich minor mergers and accretion of gas along a filament can in principle be at the origin of the ring, but the lack of any evidence for a merger remnant in the MUSE data favors the second hypothesis. Moreover, the analysis of the MUSE field of view and of the surrounding environment seems to rule out the presence of companions and possible gas donors around JO171.

JO171 gives us a unique opportunity to peer into the mechanisms that drive the formation of peculiar ring galaxies and their evolution in dense environments. Our study supports a scenario in which peculiar ring galaxies can be formed through a gas accretion event along a filament, as it was already suggested for the isolated Hoag's galaxy (Finkelman et al. 2011) and polar ring galaxies (Spavone \& Iodice 2013; Jore et al. 1996). If this is the case either the ring is able to survive for several Gyr before being destroyed, or it is slowly formed, as the stellar population ages suggest. In the case of JO171, the fate of the ring is driven by the fact that, when the galaxy interacts with the cluster ICM, the gas gets stripped and the star formation in the ring is switched off, leaving an aging and fading stellar-only ring.

\section{ACKNOWLEDGEMENTS}

We thank the anonymous Referee. This work is based on observations collected at the European Organisation for Astronomical Research in the Southern Hemisphere under ESO program 196.B-0578. This work is also based on observations taken with the AAOmega spectrograph on the AAT and the OmegaCAM camera on the VLT. This work made use of the KUBEVIZ software, which is publicly available at http://www.mpe.mpg.de/ dwilman/kubeviz/. We acknowledge financial support from PRIN-INAF 2014 and from the INAF PRIN-SKA 2017 program 1.05.01.88.04. M. M. acknowledges financial support from INAF through grant PRIN-2014-14 and from the MERAC Foundation. J.F. acknowledges financial support from a UNAM-DGAPAPAPIIT IA104015 grant, Mexico. B.V. acknowledges the support from an Australian Research Council Discovery Early Career Researcher Award (PD0028506).

\section{REFERENCES}

Agertz O., Teyssier R., Moore B., 2009, MNRAS, 397, L64
Appleton P. N., Struck-Marcell C., 1996, Fundamentals Cosmic Phys., 16, 111

Bacon R., et al., 2010, Proc. SPIE, 7735, 773508

Bekki K., Kenji 1998, ApJ, 499, 635

Bellhouse C., et al., 2017, ApJ, 844, 49

Bettoni D., Galletta G., García-Burillo S., Rodríguez-Franco A., 2001, A\&A, 374, 421

Bettoni D., Mazzei P., Rampazzo R., Marino A., Galletta G., Buson L. M., 2014, Ap\&SS, 354, 83

Biviano A., et al., 2017, A\&A, 607, A81

Bournaud F., Jog C. J., Combes F., 2007, A\&A, 476, 1179

Brocca C., Bettoni D., Galletta G., 1997, A\&A, 326, 907

Brosch N., 1985, A\&A, 153, 199

Buta R., Combes F., 1996, Fundamentals Cosmic Phys., 17, 95

Buta R., Crocker D. A., 1993, AJ, 105, 1344

Cappellari M., Copin Y., 2003, MNRAS, 342, 345

Cappellari M., Emsellem E., 2004, PASP, 116, 138

Cardelli J. A., Clayton G. C., Mathis J. S., 1989, ApJ, 345, 245

Carretti E., et al., 2012, MNRAS, 430, 1414

Cen R., Pop A. R., Bahcall N. A., 2014, Proceedings of the National Academy of Sciences, 111, 7914

Chabrier G., 2003, PASP, 115, 763

Chen Y.-M., et al., 2016, Nature Communications, 7, 13269

Coccato L., et al., 2015, A\&A, 581, A65

Dopita M. A., Sutherland R. S., Nicholls D. C., Kewley L. J., Vogt F. P. A., 2013, ApJS, 208

Fasano G., et al., 2006, A\&A, 445, 805

Finkelman I., Moiseev A., Brosch N., Katkov I., 2011, MNRAS, 418,1834

Fossati M., Fumagalli M., Boselli A., Gavazzi G., Sun M., Wilman D. J., 2016, MNRAS, 455, 2028

Fritz J., et al., 2017, ApJ, 848, 132

Girardi L., Bressan A., Bertelli G., Chiosi C., 2000, A\&AS, 141, 371

Gullieuszik M., et al., 2015, A\&A, 581, A41

Gullieuszik M., et al., 2017, ApJ, 846, 27

Gunn J. E., Gott J. Richard I., 1972, ApJ, 176, 1

Haines C. P., et al., 2015, ApJ, 806, 101

Heller C. H., Shlosman I., 1996, ApJ, 471, 143

Iodice E., et al., 2006, ApJ, 643, 200

Jaffe Y. L., Smith R., Candlish G. N., Poggianti B. M., Sheen Y.-K., Verheijen M. A. W., 2015, MNRAS, 448, 1715

Jesseit R., Naab T., Peletier R., Burkert A., 2007, MNRAS, 376, 997

Johnston E. J., Merrifield M. R., Aragon-Salamanca A., Cappellari M., 2012, MNRAS, 428, 1296

Jore K. P., Broeils A. H., Haynes M. P., 1996, AJ, 112, 438

Katkov I. Y., Sil'chenko O. K., Afanasiev V. L., 2013, ApJ, 769

Kewley L. J., Ellison S. L., 2008, ApJ, 681, 1183

Lovelace R. V. E., Chou T., 1996, ApJ, 468, L25

Maccio' A. V., Moore B., Stadel J., 2006, ApJ, 636, L25

Mamon G. A., Biviano A., Boué G., 2013, MNRAS, 429, 3079

Mapelli M., Moore B., Ripamonti E., Mayer L., Colpi M., Giordano L., 2008, MNRAS, 383, 1223

Mapelli M., Rampazzo R., Marino A., 2015, A\&A, 575

Moretti A., et al., 2014, A\&A, 564, A138

Moretti A., et al., 2017, A\&A, 599, A81

Navarro J. F., Frenk C. S., White S. D. M., 1995, ApJ, 462, 563

Oman K. A., Hudson M. J., Behroozi P. S., 2013, MNRAS, 431, 2307

Owers M. S., Couch W. J., Nulsen P. E. J., 2009, ApJ, 693, 901

Peng C. Y., Ho L. C., Impey C. D., Rix H.-W., 2010, AJ, 139, 2097

Poggianti B. M., et al., 2016, AJ, 151, 78

Poggianti B. M., et al., 2017, ApJ, 844, 48

Rhee J., Smith R., Choi H., Yi S. K., Jaffé Y., Candlish G., Sánchez-Jánssen R., 2017, ApJ, 843, 128 
Rottgering H. J. A., Wieringa M. H., Hunstead R. W., Ekers R. D., 1997, MNRAS, 290, 577

Sancisi R., Fraternali F., Oosterloo T., van der Hulst J. M., 2008, A\&ARv, 15, 189

Schweizer F., Ford, W. Kent J., Jederzejewski R., Giovanelli R., 1987, ApJ, 320, 454

Sil'chenko O. K., Moiseev A. V., 2006, AJ, 131, 1336

Smith R. J., et al., 2010, MNRAS, 408, 1417

Spavone M., Iodice E., 2013, MNRAS, 434, 3310

Spavone M., Iodice E., Arnaboldi M., Gerhard O., Saglia R., Longo G., 2010, ApJ, 714, 1081

Storey P. J., Zeippen C. J., 2000, MNRAS, 312, 813

Thakar A. R., Ryden B. S., Jore K. P., Broeils A. H., 1996, ApJ, 479,702

Tonnesen S., Bryan G. L., van Gorkom J. H., 2007, ApJ, 671, 1434

Tremonti C. A., et al., 2004, ApJ, 613, 898

Vazdekis A., Sánchez-Blázquez P., Falcón-Barroso J., Cenarro A. J., Beasley M. A., Cardiel N., Gorgas J., Peletier R. F., 2010, MNRAS, 404, 1639

Vikhlinin A., Markevitch M., Murray S. S., 2001, ApJ, 551, 160

Yoon H., Chung A., Smith R., Jaffé Y. L., 2017, ApJ, 838, 81

This paper has been typeset from a $\mathrm{T}_{\mathrm{E}} \mathrm{X} / \mathrm{LAT}_{\mathrm{E}} \mathrm{X}$ file prepared by the author. 